\title{
The Effects Of Statement Persuasiveness, Statement Strength, And Regulatory Focus On Manipulative Intent Inference
}

\author{
Sultan Alaswad Alenazi, Ph.D., King Saud University, Saudi Arabia
}

\begin{abstract}
The current research investigates consumers' perception of information when no influence attempt is perceived. Results indicate that presenting information in a non-persuasive form, such as a warning statement, results in lowering manipulative intent inferences, but only as long as the statement is not strong. Moreover, a difference in manipulative intent inference as a result of statement persuasiveness and statement strength exists only when consumers are promotion focused. Additionally, the current results demonstrate that inference of manipulative intent has a negative effect on perceived quality.
\end{abstract}

Keywords: Product Quality; Persuasiveness, Regulatory Focus

\section{INTRODUCTION}

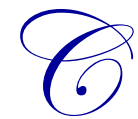

aution: the beverage you are about to consume is hot." How might this statement differ from another statement such as, "Enjoy our hot coffee." in affecting consumers' perceptions of beverage quality? Addressing this question is the objective of the current research. The latter statement is persuasive, while the former is not. Persuasion literature has focused on the effect of persuasive motives on consumers' perceptions of the message and the message provider. When people perceive an attempt to influence them, they tend to seek the best way to react to such attempt. This reaction depends on persuasion knowledge, which is consumers' accumulated knowledge of the way of perceiving and coping with influence attempts (Friestad and Wright 1994). Perception of the existence of a persuasive motive was found to lead to inferring manipulative intent (Campbell 1995). Thus, the question that results from this argument is that "Will a lack of persuasive motive lower tendencies to infer manipulative intent even in an ordinary persuasion context such as a product label?"

Information can be presented on a product label as a persuasive statement or as a non-persuasive statement. A persuasive statement is presented to convince consumers of a claim about a product characteristic. An example of such information is a claim of product quality on a product label. A non-persuasive statement is presented for a purpose other than convincing consumers of a claim about a product characteristic. An example of this statement is a warning statement on a product label.

The current research examines the effect of presenting information in a persuasive or a non-persuasive form on consumers' perception of the information as well as its provider. We suggest that a non-persuasive statement would not stimulate consumers to infer manipulative intent to the extent that a persuasive statement would. Further, we suggest that this difference in tendencies to infer manipulative intent would hold true only when the statement is weak. When a statement is strong, it will not matter if it is persuasive or non-persuasive in terms of stimulating consumers to infer manipulative intent because the statement strength is an important factor in affecting manipulative intent inference. Additionally, we argue that consumers' regulatory focus plays a role in the effect of statement persuasiveness and statement strength on manipulative intent inference such that only promotion focused consumers will be affected by statement persuasiveness and strength. This is because prevention focused consumers pay more attention to and make more attempts to avoid manipulating than do promotion focused consumers. 
This research has implications for theory and practice. Theoretically, this research contributes to the literature of persuasion since it is the first study that examines how a non-persuasive statement might differ from a persuasive statement in affecting consumers' tendencies to infer manipulative intent. Furthermore, this study sheds light on how statement strength, statement persuasiveness, and consumers' regulatory focus might interact in affecting consumers' tendencies to infer manipulative intent. For practitioners, the results of this study will help marketers enhance the persuasiveness of their communication by considering the various persuasiveness forms and different strengths in which product label statements might be presented.

\section{THEORETCAL BACKGROUND}

The Persuasion Knowledge Model (Friestad and Wright 1994) explains behavior when people perceive themselves as a target of an influence attempt. The model proposes that when facing an influence attempt, people tend to access persuasion knowledge to help them make inferences about the underlying motive of the influence agent. When they perceive a manipulative motive behind an agent's influence attempt, the influence agent is perceived negatively.

For business organizations, understanding consumers' perception of communications is important because such perception affects consumers' reactions. For example, even an organization's social activities are not taken directly by consumers as a service to the society if an ulterior motive is perceived.

In persuasion knowledge literature, researchers have focused on the effects of perception of an ulterior motive behind the information and its providing agent. One consequence of using persuasion knowledge is the inference of manipulative intent (Campbell 1995). In her study of the effect of using attention-getting tactics on persuasion, Campbell (1995) suggests that consumers' perception of the existence of persuasion tactics leads to manipulative intent inference. Coulter, Cotte, and Moore (1999) suggest that consumers do not accept information without considering the motive that might lead marketers to convey such information. Thus, current research suggests that product label information is not accepted without questioning the motive of the marketer.

What has not been studied in persuasion knowledge research is the perception of information and its providing agent when consumers do not perceive that information is provided in order to persuade them. Current research argues that when information is not perceived as persuasive, consumers will not infer a manipulative intent even in an ordinary persuasion context such as a product label. A good example of this type of information is warning information, which might be perceived as a way of informing consumers of some potential hazardous consequence of the improper use of a product. Because such warning information is not perceived as a way of persuading, consumers are not expected to tend to look for a motive behind warning information. However, statement persuasiveness is not the only factor that affects manipulative intent inference. Another important characteristic is the strength of a statement. Researchers have found statement strength to decrease the credibility of that statement (Desmet and Le Nagard 2005).

Since persuasion is typically associated with strong claims, a strong statement causes consumers to use persuasive knowledge. In other words, a strong statement is the type of information that is associated more with persuasion. Therefore, when a statement is strong, consumers tend to infer manipulative intent regardless of the statement's persuasiveness. However, the way in which a weak statement is presented determines the extent to which a manipulative intent is inferred. Thus,

H1: There will be a two-way interaction effect of statement strength and statement persuasiveness on manipulative intent inference such that: (A) When a statement is weak, presenting information in a nonpersuasive form will lower the tendencies of manipulative intent inference and (B) When a statement is strong, presenting information in a persuasive or non-persuasive form will not affect the tendencies of manipulative intent inference. 


\section{Regulatory Focus and Manipulative Intent Inference}

According to self-discrepancy theory (Higgins 1987), people have two types of goals: ideal goals relate to hope and aspiration and ought goals relate to duties and obligations. Regulatory focus theory (Crowe and Higgins 1997) suggests that ideal goals are promotion focused and ought goals are prevention focused. According to Crowe and Higgins (1997), promotion focused people are driven by growth and development needs, while prevention focused people are driven by security needs. In persuasion knowledge research, Kirmani and Zhu (2007) suggest that prevention focused consumers are more sensitive to persuasion tactics than promotion focused consumers are because the former were more reactive to negative information. In a similar way, current research expects inferring manipulative intent as a result of statement strength and statement persuasiveness to hold true only when consumers are promotion focused. This is because prevention focused consumers tend to avoid negative consequences more than promotion focused consumers do so that non-persuasive statements are not expected to lower prevention focused consumers' tendencies to infer manipulative intent. Thus,

H2: There will be a three-way interaction effect of regulatory focus, statement strength, and statement persuasiveness on manipulative intent inference such that statement strength and statement persuasiveness will interact only when consumers are promotion focused.

\section{Manipulative Intent and Perceived Quality}

A wealth of research has focused on the effects of manipulative intent and found manipulative intent to lower brand evaluations (Campbell 1995; Kirmani and Zhu 2007). Likewise, we propose that

H3: Manipulative intent will lower perceived quality.

\section{STUDY ONE}

The objective of study one was to examine the effect of persuasiveness and strength of a statement on manipulative intent inference. Study one also aimed at examining the effects of manipulative intent inference on perception of quality. The study utilized a 2 (statement persuasiveness: persuasive vs. non-persuasive) x 2 (statement strength: strong vs. weak) between subjects design. One hundred seventeen consumers participated in this study.

\section{Method}

Manipulation

Participants viewed an ad for a drain-cleaning product. In the strong persuasive condition, participants read "Our cleaner is effective in $90 \%$ of clogged drains," while in strong non-persuasive condition, participants read "Caution: Because our cleaner is $90 \%$ concentrated, gloves must be used while handling and using the cleaner." In the weak persuasive condition, participants read "Our cleaner is effective in $50 \%$ of clogged drains," while in the weak non-persuasive condition, participants read "Caution: Because our cleaner is 50\% concentrated, gloves should be used while handling and using the cleaner." Forty-three undergraduate students in an introductory marketing class participated in the pretest designed to assess the perception of statement persuasiveness and statement strength. Results indicated that persuasiveness manipulation was successful. Participants in the persuasive statement condition rated statement persuasiveness higher than did participants in the non-persuasive statement condition ( $\mathrm{M}$ persuasive $=4.36, \mathrm{M}$ non-persuasive $=2.86, \mathrm{~F}(1,41)=6.95 \mathrm{p}<.05)$. Results also revealed that statement strength manipulation was successful as participants in the strong statement condition rated statement strength higher than did participants in the weak statement condition $(\mathrm{M}$ strong $=4.24$, $\mathrm{M}$ weak $=2.83, \mathrm{~F}(1,41)=6.28 \mathrm{p}<.05)$.

\section{Measures}

Perceived quality was measured using three seven-point items scale. The first item was adjusted from Dodds, Monroe, and Grewal (1991) and the last two items from Purohit and Srivastava (2001). "This cleaner should be of (very poor to very high quality)"; "My overall impressions of the cleaner are (very bad to very good)"; "The 
cleaner is most likely going to be of high quality" (strongly disagree to strongly agree). Manipulative intent was measured using a seven-point scale adjusted from Campbell (1995). "The cleaner company intends to take advantage of customers" (strongly disagree to strongly agree).

\section{Manipulation Check}

To check manipulation of statement persuasiveness, respondents answered the following statement: "The ad tried to persuade me that the above cleaner is of high quality" (strongly disagree to strongly agree). To check manipulation of statement strength, participants responded to the following statement: "The ad made a strong claim that the above cleaner is of high quality" (strongly disagree to strongly agree).

\section{Results}

\section{Manipulation checks}

Statement persuasiveness and statement strength manipulations were successful. Participants in the persuasive statement condition perceived the statement to be more persuasive than did participants in the nonpersuasive statement condition $(\mathrm{M}$ persuasive $=4.35$, non-persuasive $=2.79 ; F(1,115)=22.29, p<.001$ ) Participants in the strong statement condition perceived the statement to be stronger than did participants in the weak statement condition $(\mathrm{M}$ strong $=4.15, \mathrm{M}$ weak $=3.13 ; F(1,115)=9.42, p<.005)$.

\section{Hypotheses Testing}

A two-way ANOVA revealed that the interaction between statement strength and statement persuasiveness had a significant effect on manipulative intent inference $(F(1,113)=8.46, p<.005)$. In the weak statement condition, inferred manipulative intent was lower when the statement was presented as a non-persuasive statement than when it was presented as a persuasive statement $(\mathrm{M}$ persuasive $=4.28, \mathrm{M}$ non-persuasive $=2.0 ; F(1,113)=$ $29.10, p<.001)$. However, in the strong statement condition, inferred manipulative intent was not significantly different in the persuasive and non-persuasive statements conditions $(\mathrm{M}$ persuasive $=4.42, \mathrm{M}$ non-persuasive $=$ $3.96, F(1,113)<1, p$ NS). These results provide support for Hypotheses 1a and $1 \mathrm{~b}$.

Consistent with Hypothesis 3, a regression analysis showed that manipulative intent had a negative effect on perceived quality $(\beta=-.202, t=-2.43, p<.05)$.

\section{STUDY TWO}

The objective of study two was to examine the effect of regulatory focus on the relationship between statement strength and statement persuasiveness in affecting manipulative intent inference. This study also aimed at examining the effects of manipulative intent inference on perceived quality. The study utilized a 2 (statement persuasiveness: persuasive vs. non-persuasive) x 2 (statement strength: strong vs. weak) x 2 (measured regulatory focus: prevention vs. promotion) between subjects design. One hundred ninety six consumers participated in this study. The procedure and manipulation were similar to those of study one with an additional variable: regulatory focus.

\section{Method}

\section{Measurement}

Perceived quality and manipulative intent were measured using the same scales as in study one. Regulatory focus was measured using one item adopted from Chatterjee, Kang, and Mishra (2005): "What do you think is more important to you, (making sure that I get what is good for me or making sure that I avoid what is bad for me)." 


\section{Results}

\section{Manipulation Check}

Statement persuasiveness and statement strength manipulations were successful. A persuasive statement was rated to be more persuasive than a non-persuasive statement $(\mathrm{M}$ persuasive $=4.40, \mathrm{M}$ non-persuasive $=3.44 ; F$ $(1,194)=12.88, p<.001)$. Additionally, a strong statement was rated to be stronger than a weak statement. (M strong $=4.27$, M weak $=3.33 ; F(1,194)=13.83, p<.001)$.

\section{Hypotheses Testing}

A three-way ANOVA with manipulative intent as a dependent variable revealed a significant interaction between statement persuasiveness, statement strength, and regulatory focus $(F(1,188)=8.83, p<.005)$. Hypothesis 2 predicted that statement persuasiveness and statement strength would interact to affect manipulative intent only when consumers were promotion focused. Consistent with Hypothesis 2, statement persuasiveness and statement strength interaction was significant when consumers were promotion focused $(F(1,188)=11.88, p<.001)$. However, when consumers were prevention focused, statement persuasiveness and statement strength interaction was not significant $(F(1,88)<1, p$ NS). Moreover, in a weak statement condition, a non-persuasive statement led promotion focused consumers to infer less manipulative intent than did a persuasive statement $(\mathrm{M}$ persuasive $=5.29$, $\mathrm{M}$ non-persuasive $=3.37 ; F(1,188)=12.11, p<.001)$. However, in the strong statement condition, inferred manipulative intent was not significantly different in the persuasive and non-persuasive statements $(\mathrm{M}$ persuasive $=$ $5.25, \mathrm{M}$ non-persuasive $=4.37 ; F(1,188)=2.01, p \mathrm{NS})$. This result provides support for Hypotheses $1 \mathrm{a}$ and $1 \mathrm{~b}$.

Consistent with Hypothesis 3, regression results revealed a negative effect of manipulative intent on perceived quality $(\beta=-.3 .75, t=-5.11 p<.005)$.

\section{CONCLUSION}

Consumers' use of persuasion knowledge to make inferences about information and its provider has gained the attention of consumer behavior researchers. The negative effect of persuasion knowledge use on information and information providers is well documented in the literature. Inference of manipulative intent is one consequence of the use of persuasion knowledge. When people perceive an influence attempt, they develop negative perceptions of information and information providers. No previous study, however, has examined consumers' perception of information when no influence is perceived.

Results of the current research indicate that consumers tend to infer less manipulative intent when no influence attempt is perceived. In addition, this research indicates that presenting information in a non-persuasive form such as a warning statement lowers manipulative intent inferences, but only as long as the statement is weak. On the other hand, when the statement is strong, persuasive and non-persuasive statements have the same effect on manipulative intent inference and statement strength exists only on consumers who are promotion focused, not on prevention focused consumers. The current results also demonstrate that inference of manipulative intent has a negative effect on perceived quality.

The current research adds to the literature in several ways. In terms of theory, this is the first study that examines consumers' perception of information when no influence attempt is perceived. For practitioners, these results suggest that consumers perceive a product's claim that is presented in a non-persuasive form more favorably.

This research, however, has some limitations. First, warning information is only one form of nonpersuasive statements. Future research should examine other forms of non-persuasiveness on the use of persuasion knowledge. Second, the product label was the context in which this research was conducted. Future research might examine the current hypotheses in other contexts, such as advertising. 


\section{ACKNOWLEDGEMENTS}

The author would like to thank The Research Center of the College of Business Administration, King Saud University, Saudi Arabia for providing support for this research.

\section{AUTHOR INFORMATION}

Dr. Sultan Alaswad Alenazi is an Assistant Professor in the Department of Marketing, College of Business Administration and Vice Dean for Educational Affairs and Programs, Prince Salman Entrepreneurship Institute at King Saud University, Saudi Arabia. His research areas include consumers' perception and behavior, promotion, and advertising. E-mail: sualenazi@ksu.edu.sa

\section{REFERENCES}

1. Campbell, M. C. (1995). When attention-getting advertising tactics elicit consumer inferences of manipulative intent: The importance of balancing benefits and investments. Journal of Consumer Psychology, 4,225-254.

2. Campbell, M. C. and A. Kirmani (2000), Consumers' Use of Persuasion Knowledge: The Effects of Accessibility and Cognitive Capacity on Perceptions of an Influence Agent. Journal of Consumer Research, 27 (1), 69-83.

3. Chatterjee, S., Kang, Y. S., \& Mishra, D. P. (2005).Market signals and relative preference: the moderating effects of conflicting information, decision focus, and need for cognition. Journal of Business Research, 58(10), 1362-1370.

4. Coulter, R. H., Cotte, J., \& Moore, M. L. (1999). Believe it or not: Persuasion, manipulation and credibility of guilt appeals. Advances in Consumer Research, 26, 288-294.

5. Crowe, E., \& Higgins, E.T. (1997). Regulatory focus and strategic inclinations: Promotion and prevention in decision-making. Organizational Behavior and Human Decision Processes, 69, 117-132.

6. Desmet, P., \& Le-Nagard, E. (2005). Differential effects of price-beating versus price-matching guarantee on retailers' price image. The Journal of Product and Brand Management, 14(6), 393-399.

7. Dodds,William B., Kent B. Monroe, and Dhruv Grewal (1991), Effects of Price, Brand, and Store Information on Buyers' Product Evaluations. Journal of Marketing Research, 28 (August) 307-19.

8. Friestad, Marian and Peter Wright (1994), The Persuasion Knowledge Model: How People Cope with Persuasion Attempts. Journal of Consumer Research, 21(1), 1-31.

9. Higgins, E.T. (1987). Self-discrepancy theory: A theory relating self and affect. Psychological Review, 94, 319-340.

10. Kirmani, Amna and Rui (Juliet) Zhu (2007). Vigilant Against Manipulation: The Effect of Regulatory Focus on the Use of Persuasion Knowledge, Journal of Marketing Research, 44(4), 688-701.

11. Purohit, D., and Srivastava, J. (2001). Effect of manufacturer reputation, retailer reputation, and product warranty on consumer judgments of product quality: A cue diagnosticity framework. Journal of Consumer Psychology. 10(3), 123-134. 